Tropical Journal of Pharmaceutical Research October 2021; 20 (10): 2015-2022

ISSN: $1596-5996$ (print); 1596-9827 (electronic) (C) Pharmacotherapy Group, Faculty of Pharmacy, University of Benin, Benin City, 300001 Nigeria.

\title{
Shenxiong Drop Pill exerts neuroprotective effect against focal cerebral ischemia partly via regulation of the expressions of ICAM-1 and caspase-3
}

\author{
Shuo-guo Jin ${ }^{1}$, Ze-ran Chen², Yang Zhang ${ }^{3}$, Meng-yuan Huang1, Meng Hou', \\ Ning-jing Ran ${ }^{1}$, Wei-yin Chen ${ }^{1}$, Fang Yang ${ }^{1 *}$, Xin-xia Zhang ${ }^{4 *}$ \\ ${ }^{1}$ Department of Neurology, Hospital of Chengdu University of Traditional Chinese Medicine, Chengdu 610072, ${ }^{2}$ School of \\ Medical and Life Sciences, 'Chengdu University of Traditional Chinese Medicine, Chengdu 611137, ${ }^{3}$ Department of Infectious \\ Diseases, Hospital of Chengdu University of Traditional Chinese Medicine, Chengdu 610072, ${ }^{4}$ Department of Endocrinology, \\ Hospital of Chengdu University of Traditional Chinese Medicine, Chengdu 610072, Sichuan Province, PR China
}

*For correspondence: Email: 348368834@qq.com (Fang Yang); 916zxx@163.com (Xin-xia Zhang)

Sent for review: 18 March 2021

Revised accepted: 27 September 2021

\begin{abstract}
Purpose: To investigate the effect of Shenxiong Drop Pill (SXDP) on cerebral infarction (CI) in rats, and the involvement of anti-inflammatory response in the process.

Methods: Rats were sacrificed at three different time points, viz, 24, 48 and $72 \mathrm{~h}$ after establishment of $\mathrm{Cl}$ model. Neurological deficit score (NDS) was determined using Bederson's neurological behavioral scoring method, whereas triphenyltetrazolium chloride (TTC) staining was used to show brain injury. The integrated optical density (IOD) of Nissl bodies and caspase-3-positive nerve cells were measured with Nissl staining and SP kit, respectively. The mRNA expression of intercellular adhesion molecule 1 (ICAM-1) was determined using reverse transcription-polymerase chain reaction (RT-PCR).

Results: SXDP produced neuroprotective effect at high, medium, and low doses. The infarct volumes in the high-, medium- and low-dose SXDP, and cyclophosphamide groups were significantly reduced at each time point. Different doses of SXDP significantly reduced the MRNA expression of ICAM-1 and the $I O D$ of caspase-3.

Conclusion: These results indicate that SXDP exerts neuroprotective effects against ischemic injury by negatively regulating ICAM-1/caspase-3 downstream of inflammatory and apoptosis pathways.
\end{abstract}

Keywords: Acute ischemic stroke, Neuroprotection, Inflammation, Middle cerebral artery occlusion, Caspase-3, Intercellular adhesion molecule 1

\begin{abstract}
This is an Open Access article that uses a funding model which does not charge readers or their institutions for access and distributed under the terms of the Creative Commons Attribution License (http://creativecommons.org/licenses/by/4.0) and the Budapest Open Access Initiative (http://www.budapestopenaccessinitiative.org/read), which permit unrestricted use, distribution, and reproduction in any medium, provided the original work is properly credited.
\end{abstract}

Tropical Journal of Pharmaceutical Research is indexed by Science Citation Index (SciSearch), Scopus, International Pharmaceutical Abstract, Chemical Abstracts, Embase, Index Copernicus, EBSCO, African Index Medicus, JournalSeek, Journal Citation Reports/Science Edition, Directory of Open Access Journals (DOAJ), African Journal Online, Bioline International, Open-J-Gate and Pharmacy Abstracts

\section{INTRODUCTION}

Stroke is a common disease worldwide. It is the third leading cause of death amongst human diseases, and the most common cause of disability [1]. Acute ischemic stroke, the most common type of stroke, accounts for $90 \%$ of strokes, and it is associated with high morbidity and mortality [2]. Rapid complete reperfusion is a regular technique used in animal models. However, in clinical experience, only a small percentage of stroke patients (approximately 10 
\%) achieve complete reperfusion, with timely treatment using tissue plasminogen activator (tPA) and thrombectomy. Indeed, most patients seldom achieve recanalization [3]. The main strategy used for acute stroke treatment is to find effective neuroprotective agents that protect nerve cells. Blockage of blood vessels leads to neuroinflammation which may trigger cell death. If the repair of vascular inflammation is enhanced, the adverse consequences of stroke may be reduced [4]. Apoptosis is considered to be the major contributor to neuronal loss after cerebral ischemia, and it is related to the enlargement of infarct areas [5].

A lot of research effort has been made in order to understand the implications of neuroinflammation and apoptosis in stroke [4]. Studies have been carried out on various treatments for reducing the risk of stroke via reductions in inflammation and apoptosis, and further research is still ongoing.

Shenxiong Drop Pill (SXDP) is comprised of Astragalus saponins, total salvianolic acid, and volatile oils from Ligusticum chuanxiong. It has a positive effect on acute ischemic stroke, as indicated in previous studies [6,7]. Intercellular adhesion molecule-1 (ICAM-1) plays a key role in the accumulation of granulocytes after focal cerebral ischemia. Deficiency of ICAM-1 reduces impairment of microcirculation and infarct size after cerebral ischemia [8]. Caspase-3 protein is a major indicator of apoptosis. Inhibition of caspase-3 activity reduces infarct size [5]. Despite what is already known, only few studies have been conducted on the dynamics of ICAM1 and caspase- 3 in ischemic stroke.

In this study, the relationship between ICAM-1 and caspase- 3 in the penumbra at 24, 48 and 72 $\mathrm{h}$ post-permanent ischemic stroke, was investigated. The findings may provide new treatment strategy for ischemic stroke.

\section{EXPERIMENTAL}

\section{Animals}

Male adult SD rats weighing 250 - $300 \mathrm{~g}$ were obtained from the Animal Experimental Center of Chengdu University of TCM. The rats were raised under standard conditions before and after surgery in the Time Biology Laboratory of Chengdu University of TCM, at room temperature range of $23-25^{\circ} \mathrm{C}$, and were fed normally for 7 days to adapt them to the laboratory environment. Thereafter, the rats were randomly assigned to seven groups: normal control, sham-operated, model and cytoxan groups, as well as high-dose SXDP, medium- dose SXDP, and low-dose SXDO groups. The study was approved by the Laboratory Animal Welfare and Ethics Committee of the Chengdu University of TCM, China (approval no. 2016-13). All experimental procedures involving rats were undertaken according to the principles of Laboratory Animal Care and the Guide for the Care and Use of Laboratory Animals, as published by the National Science Council, China. Fresh feed and water were provided ad libitum to all animal groups.

\section{Drugs and reagents}

Shenxiong Drop Pill (SXDP) was produced by the Pharmacy School of Chengdu University of TCM (Patent no. ZL200910262686, China) as a suspension at a concentration of $2 \mathrm{~g} / \mathrm{mL}$. The rats received SXDP via gavage administration of the suspension prior to MCAO surgery. The intragastric doses for the SXDP groups were determined in line with the TCM clinical dosing guidelines $(1 \mathrm{~g} / \mathrm{kg})$. In this guideline, the highdose was 20 times the clinical dose; the medium dose was ten times the clinical dose, and the low-dose was five times the clinical dose. The drug was administered intragastrically 8 days before MCAO surgery. At the same time, the cyclophosphamide group received intragastric administration of SXDP suspension at a dose of $15 \mathrm{mg} / \mathrm{kg}$ (the dose was based on the minimum dose for body surface area, and the coefficient for 200-g rat was 0.018). The control, shamoperated and MCAO groups were administered equivalent volumes of normal saline daily, in place of SXDP. The other reagents used, and their sources (in parenthesis) were: cytoxan (HC8239, BioBomei, China); caspase-3 p20 goat polyclonal IgG (sc1226, Santa Cruz, USA), TRIzol Reagent ICAM-1 (sc-71303, Santa Cruz, USA); TTC (ST2335, BioBomei, China); hematoxylin solution (C200301, Best Sun, Zhuhai, China); eosin in alcohol solution (C200403, Best Sun, Zhuhai, China), and methylene blue trihydrate (190410, Regal Biology, Shanghai, China).

\section{Establishment of MCAO rat model}

The rats were subjected to MCAO as indicated previously [6]. The rats were anesthetized with 2 \% sodium pentobarbital (F200209150; Shanghai Chemical Reagent Company, China). Then, each rat was placed in a supine position, with the limbs were fixed. The internal and external carotid arteries were gradually separated and threaded separately. The distal end of the external carotid artery was tied and threaded at the proximal end. The spare lines of the common and internal carotid arteries were gently pulled to 
temporarily block blood flow. A $5-8 \mathrm{~mm}$ cut was made from the bifurcation of the external carotid artery, and a fishing line was inserted into the cut and into the internal carotid artery through the bifurcation of the common carotid artery, to lengths of $18-20 \mathrm{~mm}$, and the entrance of the MCA was deemed blocked when resistance was felt. The proximal end of the external carotid artery was ligated, and the internal carotid artery suture was drawn out to stop bleeding. Then, the incision was sutured layer-by-layer.

\section{NDS test}

In accordance with Bederson method [10], the neurological deficit score was determined and recorded $24 \mathrm{~h}$ after the establishment of the MCAO model. The scoring criteria were as follows: $0=$ no symptoms of nervous system damage; $1=$ moderate forelimb flexion; $2=$ leaning to the paralyzed side when walking; $3=$ falling to the opposite side of the lesion; and $4=$ inability to walk alone. The people who conducted the tests and analyses were blinded to the various treatments.

\section{Sample processing}

After the neurologic deficit score was established, the rats were euthanized via intraperitoneal injection of $2 \%$ pentobarbital sodium at 24,48 and $72 \mathrm{~h}$ after the establishment of MCAO, and the brains of rats in each group were excised. The brain samples were divided into two parts: one part was used for the measurement of cerebral infarction volume, while the other was fixed in $4 \%$ paraformaldehyde, embedded in paraffin, and sliced for hematoxylin-eosin (H \& E) staining.

\section{$H$ \& E staining}

Deparaffinized sections were left in hematoxylin solution for $12 \mathrm{~min}$, washed with domestic water for $5 \mathrm{~min}$, and differentiated with $1 \%$ hydrochloric acid solution for $30 \mathrm{sec}$. Then, the sections were washed with domestic water for 5 min, blued with $1 \%$ ammonia water for $10 \mathrm{sec}$, rinsed again with domestic water for $20 \mathrm{~min}$, and placed in $95 \%$ alcohol for $3 \mathrm{~min}$. Then, the sections were fixed in $1 \%$ eosin in alcohol for 5 min. Thereafter, the sections were dehydrated in alcohol gradient (95\% alcohol for $2 \mathrm{~min}$, anhydrous alcohol $\mathrm{I}$ for $2 \mathrm{~min}$, and anhydrous alcohol II for $2 \mathrm{~min}$ ), followed by clearing in xylene I for $5 \mathrm{~min}$, and in xylene II for $5 \mathrm{~min}$. The transparent sections were mounted on glass slides using neutral gum, and covered with cover slips prior to drying in a $37^{\circ} \mathrm{C}$ oven.

\section{Determination of cerebral infarction volume}

Triphenyltetrazolium chloride (TTC) staining was used for measurement of cerebral infarction volume. The rat brain was frozen at $-20^{\circ} \mathrm{C}$ for 15 min and sectioned into 2-mm slices which were placed in $2 \%$ TTC solution at $37^{\circ} \mathrm{C}$ for $20 \mathrm{~min}$, and fixed with $4 \%$ paraformaldehyde for $24 \mathrm{~h}$. The principle of this test is that TTC chemically reacts with mitochondrial respiratory enzymes to produce a bright red color that contrasts clearly with the color of the infarct. The Image-pro Plus 6 software was used to analyze digital images of the TTC-stained brain slices.

\section{NISSL staining of rat brain sections}

Brain sections were used for Nissl staining with methylene blue trihydrate. The images were taken using Olympus image system (IX-B50, Olympus Co. Ltd., Japan), while Image-pro Plus 6 software was used for semi-quantitative analysis of the Nissl staining results at a magnification of 200. The IOD of Nissl body staining was measured in unit visual field in the normal, sham-operated and model groups; in the different SXDP-dose groups, and in the cyclophosphamide group. Five IOD values that expressed the strongest visual field for each specimen, were used as the measured values of each sample.

\section{Expression of ICAM-1 mRNA}

Real time-PCR was used to determine the expression of ICAM-1 mRNA. Total RNA was extracted from the brain tissue using TRIzol single-step method, and the RNA was preserved at $-80^{\circ} \mathrm{C}$. After the extraction of total RNA from brain tissue sections, agarose gel electrophoresis was used to purify it, and the RNA was visualized under UV light. The primers for ICAM-1 and glyceraldehyde-3-phosphate dehydrogenase (GAPDH) were synthesized by PCR primer design and synthesis. The RT-PCR was performed, with GAPDH as the internal reference gene. The sequences of the primers used are shown in Table 1.

Table 1: Primer sequences used for RT-PCR

\begin{tabular}{llll}
\hline Gene & Upstream primer sequence 5'-3' & Downstream primer sequence 5'-3' & Length (bp) \\
\hline ICAM-1 & 5'-GGGTTGGACTAACTGGATGA-3' & 5'-GGATCGAGCTCCACTCGCTC-3' & 181 \\
GAPDH & 5'-CGGAGTCAACGGATTTGGTCGTAT-3' & 5'-AGCCTTCTCCATGGTGAAGAC-3' & 306 \\
\hline
\end{tabular}


The DNA content was density-scanned using the gel imaging analysis system, and the area under the corresponding GAPDH scan curve was standardized to represent the relative expressions of ICAM-1 mRNA. Total RNA (1 ug) was used in a $50 \mathrm{uL}$ reaction system. The PCR conditions were as follows: cDNA synthesis and pre-denaturation involved one cycle of reverse transcription at $50^{\circ} \mathrm{C}$ for $30 \mathrm{~min}$, and at $94^{\circ} \mathrm{C}$ for $2 \mathrm{~min}$. The PCR amplification was carried out in 29 cycles, including denaturation at $94{ }^{\circ} \mathrm{C}$ for $30 \mathrm{sec}$, annealing at $59^{\circ} \mathrm{C}$ for $30 \mathrm{sec}$, elongation at $72{ }^{\circ} \mathrm{C}$ for $30 \mathrm{sec}$, and a final extension step at $72{ }^{\circ} \mathrm{C}$ for $10 \mathrm{~min}$. The ICAM-1 can amplify a cDNA fragment with a length of $181 \mathrm{bp}$, while GAPDH can amplify a cDNA fragment with a length of $306 \mathrm{bp}$.

\section{Caspase-3 staining of rat brain tissue sections}

The SP method was used to display caspase-3. The sections were deparaffinized for $15 \mathrm{~min}$, and rinsed twice in distilled water and $0.02 \mathrm{M}$ PBS. Then, the sections were cooled at room temperature for $15-20 \mathrm{~min}$ and rinsed three times in $0.02 \mathrm{M}$ PBS. Ten percent normal goat serum (diluted in 1:20 PBS) was used to mount the slides, which were incubated for $20 \mathrm{~min}$ in a humidified cabinet at $37{ }^{\circ} \mathrm{C}$. Filter paper was used to remove the blocking agent, followed by addition of $50 \mu \mathrm{L}$ goat anti-rat polyclonal antibody (1:100 dilution) and incubation at $37^{\circ} \mathrm{C}$ for 60 min. Thereafter, the slides were rinsed thrice with 0.02 M PBS, after which they were incubated with the secondary antibody i.e., biotin-labeled rabbit anti-goat $\lg$, for $30 \mathrm{~min}$ in a humid box at $37^{\circ} \mathrm{C}$, and rinsed three times with $0.02 \mathrm{M}$ PBS (each rinse for $5 \mathrm{~min}$ ). Second-generation horseradish enzyme-labeled streptavidin was added, and the slides were incubated for $30 \mathrm{~min}$ in a humid chamber at $37^{\circ} \mathrm{C}$, and rinsed three times with $0.02 \mathrm{M}$ PBS. The samples were then stained with $\mathrm{DAB}$, counterstained with hematoxylin, dehydrated, permeabilized, fixed and observed under a light microscope. The images were taken using Olympus image system (IX-B50, Olympus Co. Ltd., Japan), while Imagepro Plus 6 software was used for semiquantitative analysis of the caspase-3 staining results, at a magnification of 200 .

The IOD of caspase-3 staining was measured in unit visual field in the normal, sham, model and cyclophosphamide groups, as well as in the different SXDP-dose groups. Five fields with the strongest expression were selected from each specimen section, and the IOD of caspase-3positive nerve cells in each field was measured. The IOD value of positive cells in five fields of each specimen was taken as the measured value of the specimen.

\section{Statistical analysis}

The data are presented as mean \pm SD, and were statistically analyzed using SPSS 22.0 software. Differences between and amongst the groups were analyzed using one-way analysis of variance (ANOVA). Values of $p<0.05$ were considered statistically significant.

\section{RESULTS}

\section{Effect of SXDP on histopathology of brain tissue}

The neurons in the normal group and the sham operation group had clear cellular outlines, and they were arranged closely, with intact cellular morphologies. In the model group, varying sizes liquefaction necrotic areas were seen in the cerebral cortex, with neurons disappearing in the areas of necrosis. Moreover, pyknotic nuclei were visible at the edge of some infarcted areas. Edematous cells, fuzzy cell outlines, severe cell deformation, and varying degrees of inflammatory cell infiltration were also observed. However, these abnormalities were markedly alleviated in the SXDP and cytoxan groups (Figure 1).

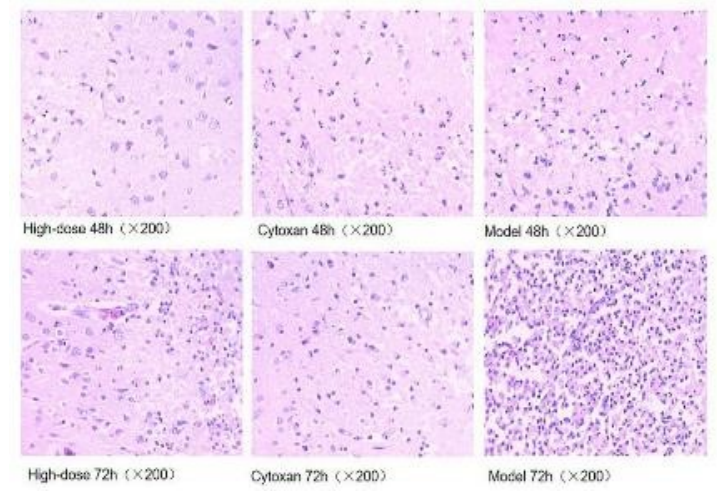

Figure 1: Effect of SXDP pretreatment on cerebral cortical histopathology in rats, as determined using $\mathrm{H}$ \& E staining

\section{SXDP pretreatment reduced the area of cerebral infarction}

The brain tissue specimens of the normal and sham operation groups showed no infarcts. However, as shown in Figure 2, compared with the model group, the infarct volumes at each time point at 24,48 and $72 \mathrm{~h}$ in the high-dose SXDP, medium-dose SXDP, low-dose SXDP and cytoxan groups, were significantly reduced $(p<$ 
0.05). Moreover, the cerebral infarction volume at 48 and $72 \mathrm{~h}$ were markedly higher than that at 24 $\mathrm{h}(p<0.05)$. The treatment with SXDP reduced cerebral infarction volume in a dose-dependent fashion $(p<0.05)$. The results for the cyclophosphamide group and SXDP high-dose group were similar $(p>0.05)$ (Figure 2$)$.

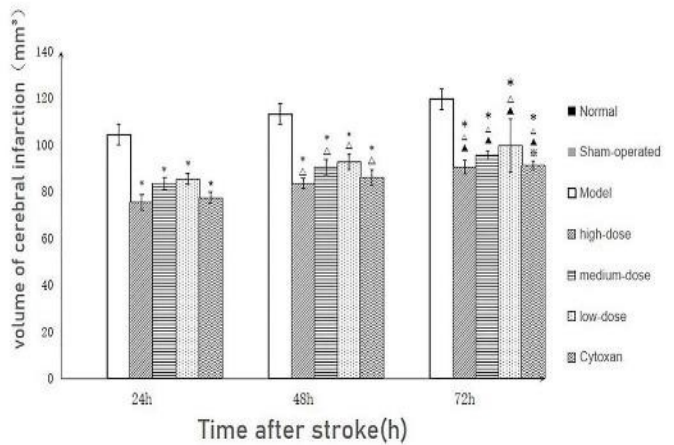

Figure 2: Effect of SXDP pretreatment on cerebral infarction volume of each group. Results are expressed as mean $\pm S D$. ${ }^{*} P<0.05$ vs. model group at same time after stroke; $\triangle \mathrm{P}<0.05^{\circ} \mathrm{vs}$. corresponding group at $24 \mathrm{~h} ;{ }^{\Delta} \mathrm{p}<0.05 \mathrm{vs}$. corresponding group at 48 $h ; \aleph_{p}>0.05$, vs. high-dose at the same time point after stroke

\section{SXDP pretreatment increased expression of Nissl body}

The integral optical density of Nissl bodies differed significantly between the normal group and the sham-operated group $(p>0.05)$. The integral optical density of Nissl body in the model group was significantly lower than those of the normal and the sham-operated groups ( $p<$ $0.05)$. After infarction, the integral optical density of Nissl body gradually decreased with time, when compared with values at 24,48 and $72 \mathrm{~h}(p$ $<0.05)$. Compared with the normal group and the sham-operated group, the integral optical density of Nissl body in the high-dose SXDP, medium-dose SXDP, low-dose SXDP, and cytoxan groups were significantly lower $(p<$ 0.05). In contrast, treatment with SXDP resulted in dose-dependent increases in the expressions of Nissl body. However, the efficacies of the cytoxan group and SXDP high-dose group were comparable $(p>0.05$; Figure 3$)$.

\section{SXDP pretreatment reduced expression of ICAM-1}

The mRNA expression levels of ICAM-1 in the normal and sham-operated groups were comparable and very weak $(p>0.05)$. In contrast, the expression of ICAM1 mRNA in the model group increased significantly, peaked at $24 \mathrm{~h}$, and decreased at 48 and $72 \mathrm{~h}$, when compared with the normal and sham-operated groups $(p<0.05)$. However, treatment with cytoxan and different doses of SXDP led to significant reductions in the expression of ICAM1 mRNA $(p<0.05)$. A dose-dependent effect was observed with DXDP on the expression of ICAM-1 mRNA, but not with Cytoxan (Figure 4).

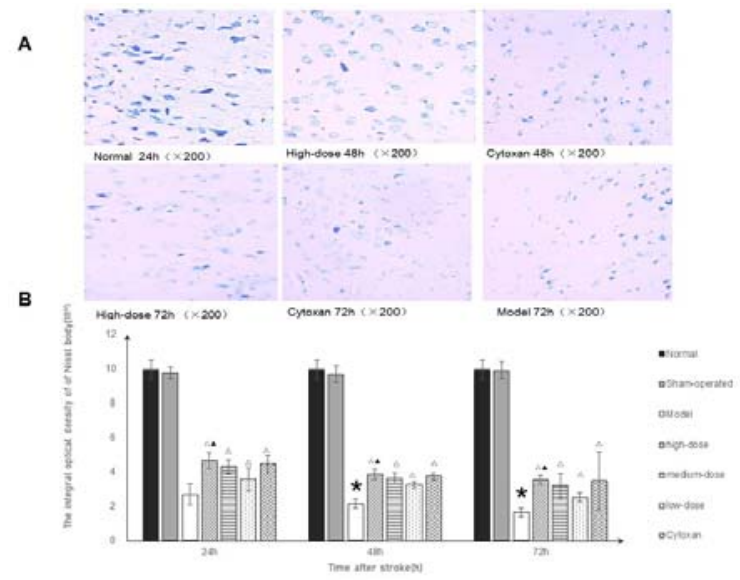

Figure 3: Effect of SXDP pretreatment on the integral optical density of Nissl bodies in each group. Values are expressed as mean $\pm \mathrm{SD}$. ${ }^{*} \mathrm{P}<0.05$, vs. model at $24 \mathrm{~h} ;{ }^{\Delta} \mathrm{p}<0.05$, vs. model group at the same time point after stroke; ${ }_{p}>0.05$, vs. cytoxan at the same time point after stroke

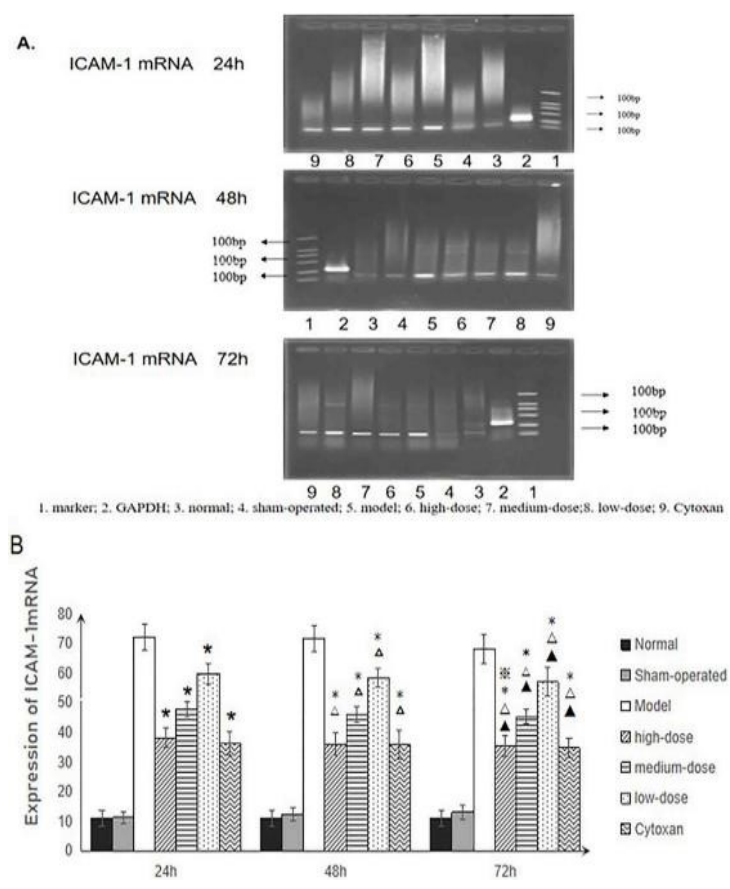

Figure 4: Effect of SXDP pretreatment on ICAM-1 mRNA expression level in each group. Values are expressed as mean $\pm S D$. ${ }^{*} P<0.05$, vs. model group at the same time point after stroke; $\Delta p<0.05$, vs. model group at $24 \mathrm{~h} ; \boldsymbol{\Delta} \mathrm{p}<0.05$, vs. model group at 48 h; ※p>0.05, vs. cytoxan group

Trop J Pharm Res, October 2021; 20(10): 2019 
SXDP pretreatment significantly inhibited the activation and expression of caspase-3

Figure 5 shows that the expression levels of caspase- 3 in the normal and sham-operated groups were comparable and very weak. However, after blocking the middle cerebral artery, caspase- 3 was activated. The IOD of nerve cell caspase- 3 was increased significantly in the model group at $24 \mathrm{~h}$, and it was gradually increased at 48 and $72 \mathrm{~h}$, with significant differences amongst values at the various time points $(p<0.05)$. However, after drug intervention, the IOD of caspase- 3 was significantly reduced, relative to the model group $(p<0.05)$. There was no significant difference in the IOD of caspase-3 between the cytoxan group and the high-dose CXDP group $(p>0.05)$.

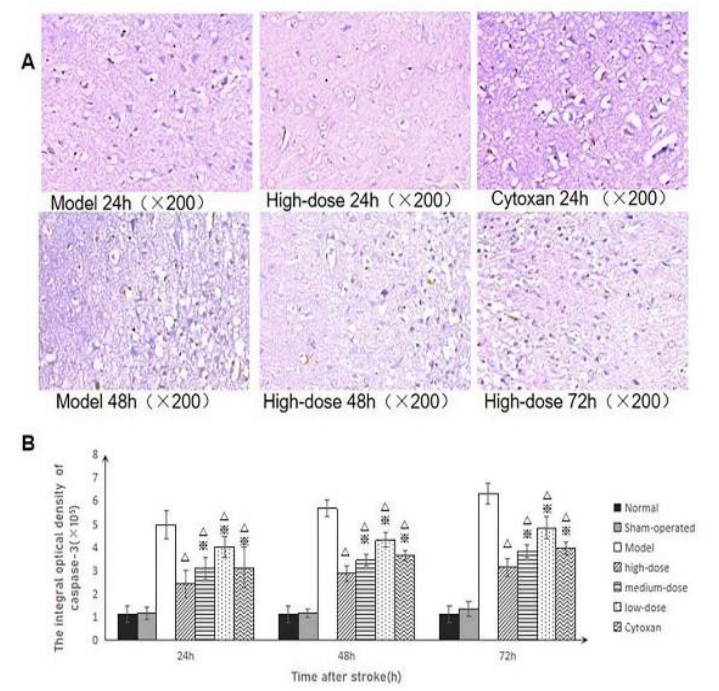

Figure 5: Effect of SXDP pretreatment on the expression levels of caspase-3 in each group. Values are expressed as mean $\pm S D$. $\triangle P<0.05$, vs. model group at the same time point after stroke; $※ p<0.05$, vs. high-dose SXDP at the same time point after stroke

\section{DISCUSSION}

Cerebral ischemia injury triggers inflammatory cascade reactions which cause the entry of peripheral immune cells into the brain tissue, thereby aggravating inflammation and cell apoptosis post-ischemia. Studies have been carried out on various treatments for reducing the risk of stroke via reduction of inflammation and apoptosis, and further research is still in progress. Leukocytes have been shown to play important roles in activation of the ischemic inflammatory cascade.

Secondary neurovascular damage to the vascular endothelial cell adhesion molecules in the brain is a key factor in the recruitment of leukocytes to inflammation sites. The most extensively studied adhesion molecule is ICAM1. Leukocytes adhere closely to endothelial cells in ischemic stroke through ICAM-1. This enhances the penetration of the blood brain barrier (BBB) by immune cells, thereby aggravating brain tissue damage which leads to cerebral edema and ischemic penumbra infarction [12]. Studies have shown that ICAM-1 expression is markedly increased by hypoxia within $4 \mathrm{~h}$ after onset of ischemia [13]. This is thought to facilitate the binding of ligands and activation of leukocytes, thereby enhancing their entry into the CNS in an inflammatory environment. Thus, ICAM-1 may be a very important effector in the regulation of the infiltration of leukocytes through the BBB in ischemic stroke. Several research groups have studied the use of ICAM-1 for preventing the recruitment of immune cells and for minimizing secondary inflammatory responses in stroke.

Apoptosis is a homeostatic mechanism that controls the population of cells. It also plays an important role after brain damage caused by ischemia and hypoxia. Apoptosis induced by ischemic stroke occurs mainly in neurons. In animal models of ischemic cerebral infarction, caspase- 3 has been identified as a key mediator of apoptosis. Research has demonstrated the upregulation of caspase- 3 in rodent and human brains after focal ischemic attack [14,15]. Moreover, the inhibition of apoptosis is an important method for preventing brain damage during ischemia $[16,17]$. It has been reported that the combination of $\mathrm{N}$-acetyl-seryl-aspartyl-lysylproline AcSDKP) and tPA significantly reduced infarct size in cerebral tissues, decreased adverse neurological outcomes, and inhibited tPA-induced leakage of aged endothelial cells, thereby down-regulating the expression of ICAM1 [18].

In the present study, RT-PCR and immunehistochemical staining were used to determine the effect of SXDP on cell apoptosis and inflammation. Moreover, the expression levels of the major indicators i.e., cleaved caspase-3 protein and ICAM-1 mRNA were measured. The results showed that ischemic infarction was significantly reduced by SXDP in the stroke model of MCAO rats, and the neurological outcomes of the rats were markedly improved. The IOD of nerve cell caspase-3 was significantly increased in the model group at $24 \mathrm{~h}$, and was slightly increased at 48 and $72 \mathrm{~h}$. The effect of high-dose SXDP was similar to that of cyclophosphamide. The inhibitory effect of high- 
dose SXDP on caspase-3 expression was stronger than that of cyclophosphamide. The mRNA expression of ICAM-1 in the model group increased significantly, peaked at $24 \mathrm{~h}$, and then decreased slightly at 48 and $72 \mathrm{~h}$.

However, SXDP decreased the expression levels of caspase- 3 mRNA and ICAM- 1 mRNA. These results show that SXDP exerted a neuroprotective effect against cerebral ischemia partly through targeted downregulation of mRNA expressions of caspase- 3 and ICAM-1. These findings may provide a new therapeutic strategy for cerebral ischemia.

Many natural compounds have been shown to be safe and beneficial for treatment of stroke. It has been reported that salvianolic acid $D$ (SaID) alleviated neurological impairment, decreased cerebral infarction, and inhibited the expressions of Bax, cytochrome $c$ and reduced caspase- 3 and caspase-9 [19]. These results also indicate that there were temporal differences in the activation status of ICAM- 1 and caspase-3: the expression of caspase-3 was increased later than that of ICAM. This suggests that therapeutic targeting of these pathways should consider their unique temporal dynamics.

It is possible that inflammation plays a different role in ischemic stroke. The dual and opposing effects of inflammatory damage and repair complicate attempts at targeting inflammatory signals in stroke patients [20]. It appears that beneficial or harmful effects of the neuroinflammatory mechanism depend on the post-cerebral ischemia period. At the early stage of ischemic stroke, inflammation may cause ischemic damage to brain tissues, while in the later stage, inflammation may be a neuroprotective effect which promotes angiogenesis and recovery, as well as plasticity of brain nerves through repair [21]. Hence, a better characterization of time-dependent effects of neuroinflammation may contribute to the development of neuroprotective strategies after stroke.

\section{CONCLUSION}

These findings indicate that SXDP negatively regulates the downstream inflammation and apoptosis pathways of ICAM-1/caspase-3, inhibits inflammatory response in the early stage of cerebral infarction in MCAO model rats, and reduces inflammatory damage after cerebral ischemia. However, the molecular pathway involved in the neuroprotective effect of SXDP requires further investigation.

\section{DECLARATIONS}

\section{Acknowledgement}

This study was supported by the National Natural Science Foundation of China (no. 82004342), the Fund of Chengdu University of Traditional Chinese Medicine (no. ZYTS2019014), and the Fund of Hospital of Chengdu University of Traditional Chinese Medicine (no. 18PY28). Special thanks go to Professor Zhuyun Yan of Chengdu University of Traditional Chinese Medicine, College of Pharmacy.

\section{Conflict of interest}

The authors declare that they have no known competing financial interests or personal relationships that could have influenced the study reported in this manuscript.

\section{Contributions of authors}

We declare that this work was done by the authors named in this manuscript, and all liabilities pertaining to claims relating to the content of this article will be borne by the authors. Shuo-guo Jin, Zeran Chen and Yang Zhang, as first co-authors, contributed equally to this work. All three of them conducted the experimental aspect of the research, performed statistical analysis, and prepared the initial draft of the manuscript. Fang Yang, Xin-xia Zhang and Wei-yin Chen conceived and designed the study. Zeran Chen and Ningjing Ran collected and analyzed the data. Shuoguo Jin, Ze-ran Chen and Yang Zhang wrote the manuscript. Xin-xia Zhang supervised the project. Mengyuan Huang and Meng Hou helped to complete the statistics form.

\section{Open Access}

This is an Open Access article that uses a funding model which does not charge readers or their institutions for access and distributed under the terms of the Creative Commons Attribution License (http://creativecommons.org/licenses/by/ 4.0) and the Budapest Open Access Initiative (http://www.budapestopenaccessinitiative.org/rea d), which permit unrestricted use, distribution, and reproduction in any medium, provided the original work is properly credited.

\section{REFERENCES}

1. GBD 2016 Stroke Collaborators. Global, regional, and national burden of stroke, 1990-2016: A systematic

Trop J Pharm Res, October 2021; 20(10): 2021 
analysis for the Global Burden of Disease Study 2016. Lancet Neurol. 2019; 18(5): 459-480.

2. Benjamin EJ, Blaha MJ, Chiuve SE, Cushman M, Das $S R$, Deo $R$, de Ferranti SD, Floyd J, Fornage $M$, Gillespie $C$, et al. Heart disease and stroke statistics2017 update: a report from the American heart association. Circulation. 2017; 135(10): e146-e603.

3. Lambertsen KL, Finsen B, Clausen BH. Post-stroke inflammation-target or tool for therapy? Acta Neuropathol Commun. 2019; 137(5): 693-714.

4. Parikh NS, Merkler AE, ladecola C. Inflammation, autoimmunity, Infection, and stroke: epidemiology and lessons from therapeutic intervention. Stroke. 2020; 51(3): 711-718.

5. Ferrer I, Planas AM. Signaling of cell death and cell survival following focal cerebral ischemia: life and death struggle in the penumbra. J Neuropathol Exp Neurol. 2003; 62(4): 329-339.

6. Jin SG, Deng JL, Yin HY, Chen ZR, Liu MJ, Sun HH, Ran $N J$, Zhang L, Yang $D D$, Chen WY. Pretreatment with Shenxiong Drop Pill induces AQP4 mediated neuroprotective effect on middle cerebral artery occlusion in rats. Trop J Pharm Res. 2020; 19 (8): 17151722.

7. Cao $P$, Chen WY, Yang F, Zhu BD, Liu FY, Xie W, Fang $Y$. Impact of Shenxiong drop pill on the size of ischemic area and expression of NF-KB in the brain of rats model with MACO. J Sichuan Univ, Med Sci Ed. 2014; 5: 801804.

8. Kitagawa K, Matsumoto M, Mabuchi T, Yagita Y, Ohtsuki $T$, Hori M, Yanagihara T. Deficiency of intercellular adhesion molecule 1 attenuates microcirculatory disturbance and infarction size in focal cerebral ischemia. J Cereb Blood Flow Metab. 1998; 18(12): 1336-1345.

9. Bederson JB, Pitts LH, Tsuji M, Nishimura MC, Davis RL, Bartkowski H. Rat middle cerebral artery occlusion: evaluation of the model and development of a neurologic examination. Stroke. 1986; 17(3): 472-476.

10. Shichita T, Sakaguchi R, Suzuki M, Yoshimura A. Postischemic inflammation in the brain. Front Immunol. 2012; 3:132.

11. Mizuma A, Yenari MA. Anti-Inflammatory Targets for the Treatment of Reperfusion Injury in Stroke. Front Neurol. 2017; 8: 467.
12. Nielsen $H H$, Soares $C B$, Høgedal SS, Madsen JS, Hansen RB, Christensen AA, Madsen C, Clausen BH, Frich $L H$, Degn $M$, et al. Acute Neurofilament Light Chain Plasma Levels Correlate With Stroke Severity and Clinical Outcome in Ischemic Stroke Patients. Front Neurol. 2020; 11: 448.

13. Yang XM, Chen L, Li Y, Gao F, Yan ZB, Zhang P, Wu JB, Wang HL, Sun BZ. Protective effect of Hydroxysafflor Yellow $A$ on cerebral ischemia reperfusion-injury by regulating GSK3ßmediated pathways. Neurosci Lett. 2020; 736: 135-258.

14. Mahale A, Kumar R, Sarode LP, Gakare S, Prakash A, Ugale RR. Dapsone prolong delayed excitotoxic neuronal cell death by interacting with proapoptotic/survival signaling proteins. J Stroke Cerebrovasc Dis. 2020; 29(8): 104848.

15. Jin B, Kim H, Choi JI, Bae HB, Jeong S. Avenanthramide C Prevents Neuronal Apoptosis via PI3K/Akt/GSK3 $\beta$ Signaling Pathway Following Middle Cerebral Artery Occlusion. Brain Sci 2020; 10(11): 878.

16. Chen XW, Yao ZJ, Xian P, Long W, Wu HC, Ou YT, Lai $J B$. Eupafolin alleviates cerebral ischemia/reperfusion injury in rats via blocking the TLR4/NFKB signaling pathway. Mol Med Rep. 2020; 22(6): 5135-5144.

17. Li C, Zhang L, Wang CY, Teng $H$, Fan BY, Chopp $M$, Zhang ZG. N-Acetyl-Seryl-Aspartyl-LysylProline Augments Thrombolysis of tPA (Tissue-Type Plasminogen Activator) in Aged Rats After Stroke. Stroke. 2019; 50(9): 2547-2554.

18. Zhang $W$, Song JK, Li W, Kong DW, Liang $Y$, Zhao $X Y$, Du GH. Salvianolic Acid D Alleviates Cerebral IschemiaReperfusion Injury by Suppressing the Cytoplasmic Translocation and Release of HMGB1-Triggered NF-KB Activation to Inhibit Inflammatory Response. Mediators Inflamm. 2020;2020: 9049614.

19. Lambertsen KL, Finsen B, Clausen BH. Post-stroke inflammation-target or tool for therapy? Acta Neuropathol. 2019; 137(5): 693-714.

20. Yang C, Hawkins KE, Doré S, Candelario-Jalil E. Neuroinflammatory mechanisms of blood-brain barrier damage in ischemic stroke. Am J Physiol Cell Physiol. 2019; 316(2): C135-C153.

Trop J Pharm Res, October 2021; 20(10): 2022 